\title{
Design, rationale, and initiation of the Surgical Interventions for Moderate Ischemic Mitral Regurgitation Trial: A report from the Cardiothoracic Surgical Trials Network
}

\author{
Peter K. Smith, MD, ${ }^{\mathrm{a}}$ Robert E. Michler, MD, ${ }^{\mathrm{c}}$ Y. Joseph Woo, MD, ${ }^{\mathrm{d}}$ John H. Alexander, MD, MHS, \\ John D. Puskas, MD, ${ }^{\mathrm{e}}$ Michael K. Parides, PhD, ${ }^{\mathrm{f}}$ Rebecca T. Hahn, MD, ${ }^{\mathrm{g}}$ Judson B. Williams, MD, ${ }^{\mathrm{a}}$ \\ John M. Dent, MD, ${ }^{h}$ T. Bruce Ferguson, Jr, MD, ${ }^{i}$ Ellen Moquete, RN, BSN, ${ }^{f}$ Eric A. Rose, MD, ${ }^{f}$ \\ Pierre Pagé, MD, ${ }^{\mathrm{j}}$ Neal O. Jeffries, PhD, ${ }^{\mathrm{k}}$ Patrick T. O'Gara, MD, ${ }^{1}$ and Deborah D. Ascheim, $\mathrm{MD}^{\mathrm{f}}$
}

Objective: Patients with coronary artery disease complicated by moderate ischemic mitral regurgitation have demonstrably poorer outcome than do patients with coronary artery disease but without mitral regurgitation. The optimal treatment of this condition has become increasingly controversial, and a randomized trial evaluating current practices is warranted.

Methods: We describe the design and initial execution of the Cardiothoracic Surgical Trials Network Surgical Interventions for Moderate Ischemic Mitral Regurgitation Trial.

Results: This is an ongoing prospective, multicenter, randomized, controlled clinical trial designed to test the safety and efficacy of mitral repair in addition to coronary artery bypass grafting in the treatment of moderate ischemic mitral regurgitation.

Conclusions: The results of the Cardiothoracic Surgical Trials Network Surgical Interventions for Moderate Ischemic Mitral Regurgitation Trial will provide long-awaited information on controversial therapies for this morbid disease process. (J Thorac Cardiovasc Surg 2012;143:111-7)

Supplemental material is available online.

Functional ischemic mitral regurgitation (IMR) can be defined as mitral valve regurgitation in the setting of ischemic heart disease without evidence of structural pathology of the

\footnotetext{
From the Divisions of Cardiovascular and Thoracic Surgery ${ }^{\mathrm{a}}$ and Cardiology, ${ }^{\mathrm{b}}$ Duke University, Durham, NC; the Department of Cardiovascular and Thoracic Surgery, ${ }^{c}$ Montefiore-Einstein Heart Center, New York, NY; the Division of Cardiovascular Surgery, ${ }^{\mathrm{d}}$ University of Pennsylvania, Philadelphia, Pa; the Division of Cardiothoracic Surgery, ${ }^{\mathrm{e}}$ Emory University, Atlanta, Ga; the International Center for Health Outcomes and Innovation Research (InCHOIR) in the Department of Health Evidence \& Policy, ${ }^{\mathrm{f}}$ Mount Sinai School of Medicine, New York, NY; the Division of Cardiology, ${ }^{\mathrm{g}}$ Columbia University Medical Center, New York, NY; the Cardiovascular Division, ${ }^{\text {h }}$ University of Virginia, Charlottesville, Va; the Department of Cardiovascular Sciences, ${ }^{\mathrm{i}}$ East Carolina Heart Institute at East Carolina University, Greenville, NC; the Department of Surgery, ${ }^{\mathrm{j}}$ Hôpital du Sacré-Cœur de Montréal, Montréal, Québec, Canada; the Office of Biostatistics Research, ${ }^{\mathrm{k}}$ National Heart, Lung, and Blood Institute, Bethesda, Md; and the Cardiovascular Division, ${ }^{1}$ Brigham and Women's Hospital, Boston, Mass.

Supported by the National Heart Lung and Blood Institute, the Canadian Institute of Health Research, and the National Institute of Neurological Diseases and Stroke (grant no. 7U01 HL088942).

Disclosures: Authors have nothing to disclose with regard to commercial support.

Received for publication Feb 16, 2011; revisions received May 13, 2011; accepted for publication May 20, 2011; available ahead of print July 26, 2011.

Address for reprints: Deborah D. Ascheim, MD, InCHOIR, Department of Health Evidence and Policy, Mount Sinai School of Medicine, One Gustave L. Levy Pl, Box 1077, New York, NY 10029 (E-mail: deborah.ascheim@mssm.edu). $0022-5223 / \$ 36.00$

Copyright (C) 2012 by The American Association for Thoracic Surgery doi:10.1016/j.jtcvs.2011.05.006
}

valve apparatus. It results from postinfarction left ventricular dysfunction and dilatation (remodeling), papillary muscle displacement with leaflet tethering, and progressive annular dilatation. ${ }^{1,2}$ It is often associated with a regional wall motion abnormality and coronary artery disease in the corresponding territory. Structural or organic mitral regurgitation, on the other hand, connotes a distinct pathology of the mitral valve, most commonly myxomatous degeneration, mitral valve prolapse, or Barlow disease. Each of these pathologic entities may lead to chronic nonischemic mitral regurgitation.

The presence of IMR is a significant predictor of adverse short- and long-term outcomes in patients with coronary artery disease, particularly after acute myocardial infarction. ${ }^{3-6}$ When coronary artery disease is treated with coronary artery bypass grafting (CABG) alone, the unadjusted incidence of death has been found to be significantly increased even in the presence of only mild IMR $(8.4 \%$ at 1 year) relative to patients with no IMR $(3.8 \%$ at 1 year). The mortality risk increases with increasing severity of mitral regurgitation and has been found to be twice as great in patients with moderate IMR treated with CABG alone $(16.9 \%$ at 1 year). ${ }^{7}$ The surgical treatment of IMR has become increasingly controversial, with approximately equal numbers of patients treated with either combined $\mathrm{CABG}$ and mitral valve repair or replacement or with $\mathrm{CABG}$ alone. ${ }^{8-10}$ Operative mortality for $\mathrm{CABG}$ as well as for $\mathrm{CABG}$ combined with mitral valve repair has declined steadily nationwide during the past 5 years ${ }^{11}$; however, the additional 


\section{Abbreviations and Acronyms \\ CABG = coronary artery bypass grafting \\ CTSN $=$ Cardiothoracic Surgical Trials Network \\ ERO = effective regurgitant orifice \\ IMR $=$ ischemic mitral regurgitation \\ LVESVI = left ventricular end-systolic volume index \\ NHLBI = National Heart, Lung, and Blood Institute \\ TEE $=$ transesophageal echocardiography \\ TTE $=$ transthoracic echocardiography}

aortic crossclamp time and cardiopulmonary bypass time associated with the performance of mitral valve repair increase the risk of the combined procedure. ${ }^{12,13}$ This approach does not allow a purely off-pump procedure. Selection of the appropriate patients is thus imperative to ensure that the trade-off of the additional risk of mitral valve repair is necessary and provides additional short- or long-term benefit.

Proponents of treating mild to moderate IMR with revascularization alone argue that revascularization improves regional contractility and restores mitral valve papillary muscle continuity, thus normalizing mitral valve function. ${ }^{14,15}$ On the other hand, proponents of a more aggressive treatment strategy cite the negative consequences of ongoing mitral regurgitation. Myocardial revascularization alone may be insufficient to restore normal ventricular physiology once mitral regurgitation has developed. Correction of mitral regurgitation may prevent progressive adverse remodeling, improve cardiac function, and attenuate the risk of heart failure.

Available evidence addressing treatment decisions for IMR is limited to small, single-center, randomized trials, observational studies, and case series, ${ }^{7-11,13,15}$ in which correction for significant and substantial imbalances in baseline patient characteristics is problematic, making it difficult to develop a clear understanding of appropriate treatment options. These studies are also limited by variable definitions of the severity and etiology of mitral regurgitation, variable surgical repair techniques, potential publication bias, limited patient follow-up, and lack of information on key secondary outcomes such as quality of life. ${ }^{12}$ Importantly, the recently published American College of Cardiology and American Heart Association guidelines both for CABG and for the management of patients with valvular heart disease avoid addressing the decision algorithm for IMR. ${ }^{16,17}$ The only consensus established from literature review is that the preferred treatment is unknown and should be individualized and that a randomized clinical trial to generate necessary evidence on which to base clinical decisions is essential. ${ }^{7,11,18,19}$

\section{TRIAL DETAILS}

In February 2004, the National Heart, Lung, and Blood Institute (NHLBI) advisory council proposed that the NHLBI evaluate the status of cardiac surgery and its future directions. The NHLBI convened a working group on future directions in cardiac surgery, which called for the formation of the Cardiothoracic Surgical Trials Network (CTSN), which was designed to develop a culture of rigorous clinical evaluative research within the field of cardiac surgery. The CTSN includes integration of both cardiologists and surgeons in the ownership of and responsibility for trials bridging the integrated specialties. Moderate IMR was the top priority for initial investigation of both the NHLBI working group and the CTSN investigators. ${ }^{18}$

\section{Trial Objectives}

The CTSN Surgical Interventions for Moderate Ischemic Mitral Regurgitation Trial seeks to evaluate the safety and efficacy of mitral valve repair for moderate IMR. Specifically, the trial compares mitral valve repair combined with $\mathrm{CABG}$ to $\mathrm{CABG}$ alone in this patient population. The primary aim of the trial is to evaluate the effects of these 2 surgical approaches on left ventricular remodeling, as assessed by left ventricular end-systolic volume index (LVESVI). Secondary aims of this trial include assessment of the effects of these 2 surgical interventions on the severity of the mitral regurgitation, regional and global cardiac performance, mortality, adverse events, quality of life, functional status, neurocognitive function, and health resource use.

\section{Trial Design}

This is a prospective, multicenter, randomized clinical trial conducted at the clinical centers participating in the CTSN (Appendix E1). Patients deemed eligible are randomly assigned in a 1:1 allocation to CABG combined with mitral valve repair or CABG alone (Figure 1). The enrollment period is estimated to be 36 months, and all patients will be followed up for 24 months after randomization. A minimum of 2 years of follow-up is intended, although the primary end point will be assessed at 1 year.

Neither patients nor investigators are blinded to the treatment assignment because of the nature of treatment intervention. The investigators are, however, blinded to all data from other clinical sites, with the exception of information required for institutional review board reporting purposes. All protocol-defined echocardiograms are analyzed by a centralized core laboratory, and all core laboratory personnel are blinded to clinical characteristics and outcomes. Serious and protocol-defined adverse events are adjudicated by an independent event adjudication committee. Trial 


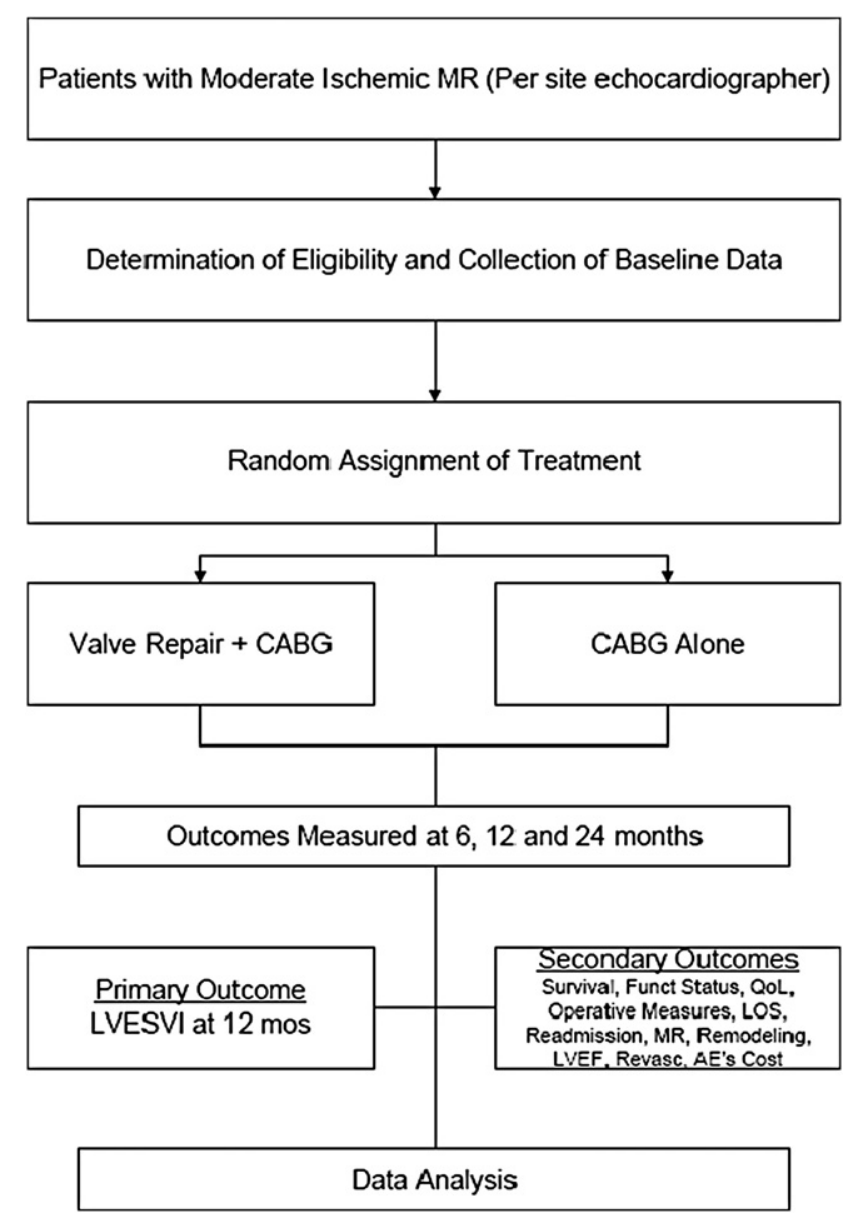

FIGURE 1. Study design flow chart. $M R$, Mitral regurgitation; $C A B G$, coronary artery bypass grafting; $L V E S V I$, left ventricular systolic volume index; $L O S$, length of stay; $L V E F$, left ventricular ejection fraction; $A E$ 's, adverse events.

oversight is provided by an independent data and safety monitoring board appointed by the NHLBI.

\section{Patient Population}

The patient population for this trial consists of patients with clinically significant coronary artery disease and an indication for CABG associated with moderate IMR. The ischemic etiology and degree of mitral regurgitation is assessed by transthoracic echocardiography (TTE) within 30 days before randomization, as determined by the clinical site echocardiographer. All clinical site laboratories are accredited by the echocardiography core lab with regard to both image acquisition and interpretation before site activation and patient enrollment. Patients are eligible to participate regardless of the presence or absence of symptoms attributable to mitral regurgitation. Furthermore, to increase the generalizability of the results of this trial, because the timing of referral for surgical intervention in patients with moderate IMR is quite variable in the community, the trial was designed in the absence of a "run-in" period that would otherwise have optimized medical therapy before the qualifying TTE. As such, patients can be included in the trial even if subsequent TTEs after the qualifying TTE, but before randomization, demonstrate a decrease in the severity of mitral regurgitation with optimal medical therapy. $\mathrm{Pa}-$ tients who have echocardiographic documentation of severe IMR by TTE at any time are candidates for a separate CTSN trial comparing mitral valve repair and replacement.

\section{Echocardiographic Assessment of the Degree of Mitral Regurgitation}

This trial is designed to evaluate a patient population within the clinical spectrum of moderate IMR, as identified by TTE. The initial mitral valve inclusion criteria were an effective regurgitant orifice (ERO) $0.2 \mathrm{~cm}^{2}$ to $0.39 \mathrm{~cm}^{2}$ in area $^{7,11,18}$ and a structurally normal mitral valve. It became clear early in the course of screening patients for enrollment, however, that the ERO area criterion was overly restrictive and led to the exclusion of patients with other semiquantitative echocardiographic measures of moderate mitral regurgitation. Review of trial screening logs indicated that failure to meet the original ERO area criterion, despite the consensus opinion of the surgical principal investigator and the site echocardiographer that the mitral regurgitation was moderate, was the major cause of screening failure. The echocardiographic inclusion criteria were therefore broadened to reflect more closely the consensus of surgeons and cardiologists in an effort to ensure that enrolled patients more closely resembled those referred in clinical practice. Specifically, inclusion was redefined as moderate IMR with (1) an ERO area of $0.2 \mathrm{~cm}^{2}$ to $0.39 \mathrm{~cm}^{2}$ or (2) an ERO area less than $0.2 \mathrm{~cm}^{2}$ and additional evidence of more than mild mitral regurgitation, as suggested by such integrative criteria as the density of the mitral regurgitation velocity profile, pulmonary vein systolic flow abnormalities, and left atrial jet area (Tables 1 and 2). ${ }^{7,11,18,20}$

\section{Eligibility Criteria}

Criteria for inclusion in this trial, beyond the echocardiographic identification of moderate IMR, are intentionally broad, focusing on targeting patients with coronary anatomy amenable to CABG and a clinical indication for revascularization. Patients are excluded from participation if they have evidence of concomitant structural mitral valve disease, previous mitral valve intervention, or planned concomitant intraoperative procedures, with the exception of maze procedures or closure of a patent foramen ovale or an atrial septal defect. The key eligibility criteria for the trial are listed in Table 3.

\section{Randomization}

Randomization is blocked and stratified by clinical center. Randomization is performed intraoperatively, after the sternotomy but before cannulation of the aorta, to minimize 
TABLE 1. Integrative method criteria for echocardiographic assessment of degree of mitral regurgitation: Echocardiographic color Doppler and quantitative criteria

\begin{tabular}{llcc}
\hline & \multicolumn{1}{c}{ Mild } & Moderate & Severe \\
\hline Color flow jet area & $<20 \%$ of LA area & $20 \%-39 \%$ of LA area & $\begin{array}{c}\text { Large central jet (usually }>10 \mathrm{~cm}^{2} \\
\text { or }>40 \% \text { of LA area) or variable } \\
\text { size wall-impinging jet swirling in LA }\end{array}$ \\
Quantitative parameters & & & $\geq 0.70$ \\
Vena contracta width $(\mathrm{cm})$ & $<0.30$ & $0.30-0.69$ & $\geq 0.40$ \\
Effective regurgitant orifice area $\left(\mathrm{cm}^{2}\right)$ & $<0.20$ & $0.20-0.39$ & \\
\hline
\end{tabular}

$L A$, Left atrium.

the likelihood of enrolling patients in the study with unexpected surgical contraindications to mitral valve repair. The timing of randomization was chosen to ensure both that cannulation for cardiopulmonary bypass is possible and that patients with structural mitral valve pathology identified by intraoperative transesophageal echocardiography (TEE) not detected on the original TTE study are excluded before randomization. The randomization process is controlled centrally and performed through a webbased data-collection system that automates the delivery of randomization codes. The treatment assignment is sent to the site coordinator electronically in a secure fashion, and electronic verification of the treatment assignment is required before proceeding with the treatment intervention. Thereafter, primary efficacy is to be analyzed by intention to treat; that is, the patients are grouped by their assignment at randomization regardless of whether they actually receive the treatment to which they are initially assigned.

\section{End Point Definitions}

The primary end point for the trial is the degree of left ventricular remodeling, as assessed by the LVESVI measured by TTE at 12 months after randomization. The principal secondary end point is a composite of major adverse cardiac events, as defined by an unweighted composite score composed of the following components: death, stroke, worsening heart failure $(\geq 1$ New York Heart Association class), hospitalization for heart failure, and mitral valve reintervention. Additional secondary end points include all-cause mortality, functional status assessed by peak volume of oxygen uptake during cardiopulmonary exercise testing, quality of life (36-item Short Form Health Survey, Duke Activity Status Index, and Minnesota Living with Heart Failure questionnaire), and health care costs.

\section{Treatment Intervention}

Procedural technique. All patients enrolled in this trial undergo CABG according to each operating surgeon's technical preference, with the exception of cardiopulmonary bypass support, which is required for all patients in the trial. For those patients randomly assigned to undergo mitral valve repair in addition to CABG, the repair is performed with standard techniques but the protocol mandates the use of a complete ring and downsizing by 2 sizes whenever possible. The other elements of operative and perioperative clinical care are not prescribed by the protocol.

Surgeon certification. To minimize the effect of variation in operator expertise for the purposes of this clinical trial, all surgeons operating on patients enrolled in the trial must meet a minimum threshold of experience and be certified by the site principal investigator. Protocol-specified surgical certification requires that the surgical investigator perform a minimum of 10 mitral valve repair procedures per year, averaged over 2 years. A certified clinical site surgeon must participate in the mitral valve procedure of a patient enrolled at that site if the primary surgeon is not certified.

\section{Follow-up}

Postrandomization data collection occurs at predetermined study visits as well as at event-driven visits. Planned study visits include the first postoperative day and postoperative months 6,12 , and 24 . Blood, urine, and tissue

TABLE 2. Integrative method criteria for echocardiographic assessment of degree of mitral regurgitation: Echocardiographic supportive criteria

\begin{tabular}{llll}
\hline Structural Doppler parameters & \multicolumn{1}{c}{ Mild } & \multicolumn{1}{c}{ Moderate } & Severe \\
\hline Left atrial size & Normal & Normal or dilated & Usually dilated \\
Left ventricular size & Normal & Normal or dilated & Usually dilated \\
Mitral leaflets or support apparatus & Normal or abnormal & Normal or abnormal & Abnormal, flail leaflet, ruptured papillary muscle \\
Mitral inflow (pulsed wave) & A wave dominant & Variable & E wave dominant (usually 1.2 m/s) \\
Jet density (continuous wave) & Incomplete or faint & Dense & Dense \\
Jet contour (continuous wave) & Parabolic & Usually parabolic & Early-peaking triangular \\
Pulmonary vein flow & Systolic dominance & Systolic blunting & Systolic flow reversal \\
\hline
\end{tabular}


TABLE 3. Selected eligibility criteria

Inclusion criteria
1. Moderate mitral regurgitation in the judgment of the clinical site
echocardiographer, as assessed by transthoracic echocardiography
with the integrative method
2. Coronary artery disease amenable to coronary artery bypass grafting
and a clinical indication for revascularization
3. Age $\geq 18$ y
4. Ability to sign informed consent and release of medical information
forms
Key exclusion criteria
1. Any evidence of structural (chordal or leaflet) mitral valve disease
2. Inability to derive effective regurgitant orifice and left ventricular
end-systolic volume index by transthoracic echocardiography
3. Planned concomitant intraoperative procedures (with exception of
maze procedure or closure of patient foramen ovale or atrial septal
defect)
4. Previous surgical or percutaneous mitral valve intervention
5. Contraindication to cardiopulmonary bypass
6. Clinical signs of cardiogenic shock at the time of randomization
7. Treatment with long-term intravenous inotropic therapy at the time of
randomization
8. ST-segment elevation myocardial infarction requiring intervention
within 7 days before randomization

sample collections are performed intraoperatively, and additional blood and urine collections are made 6 and 12 months after randomization. Physical examination, New York Heart Association functional classification, angina class determination, echocardiography, and quality of life assessment are performed at postoperative months 6, 12, and 24. Cardiopulmonary exercise testing is performed (if possible) at baseline and at 6 and 12 months. Neurocognitive testing is performed at baseline and 12 postoperative months. Direct cost data are obtained quarterly for all randomized patients. A minimum of 2 years of follow-up is intended, although the primary end point is determined at 1 year.

\section{Sample Size}

For calculation of sample size, we assumed the mean baseline LVESVI in the target population to be $80 \mathrm{~mL} / \mathrm{m}^{2}$ (normal LVESVI approximately $25 \mathrm{~mL} / \mathrm{m}^{2}$ ). For patients randomized to undergo $\mathrm{CABG}$ only, we anticipate a $5 \%$ reduction in LVESVI, or an absolute change of $4 \mathrm{~mL} / \mathrm{m}^{2}$. A clinically important additional reduction of $12 \mathrm{~mL} / \mathrm{m}^{2}$, or a total reduction of $20 \%\left(16 \mathrm{~mL} / \mathrm{m}^{2}\right)$ is estimated for patients who undergo mitral valve repair in addition to CABG. Assuming that baseline and 12 month LVESVI in both arms follow a gamma distribution with a common $\mathrm{SD}$ of $35 \mathrm{~mL} / \mathrm{m}^{2}$, a total of 300 patients, randomized with equal probability to each arm provides approximately $90 \%$ power to detect this additional difference of $12 \mathrm{~mL} / \mathrm{m}^{2}$ in LVESVI.

\section{Primary Analysis}

The primary null hypothesis will be tested in an intent-totreat analysis with a .05 level 2-tailed Wilcoxon rank sum test. The choice of the Wilcoxon rank sum test for the primary analysis was motivated by the expectation of a relatively substantial amount of nonignorable missing data, primarily as a result of patient death; 1-year mortality is expected to range from $10 \%$ to $15 \%$ and may differ between randomization arms.

\section{Additional Analyses}

The details of the bypass operation performed (number of grafts placed, quality of grafts, and target vessels) and assessment of regional and global left ventricular function at 6,12 , and 24 months will allow examination of the success of the revascularization component of the procedure. The interaction between the preoperative plan for revascularization, any variation from the plan, the resulting change in global and regional left ventricular function, and associated change in the severity of IMR will thus be evaluated. This analysis may identify patient subpopulations that differ in meeting the primary and secondary end points and are perhaps more responsive to revascularization alone.

\section{DISCUSSION}

The cardiac surgical community has not been recognized historically for the conduct of large-scale, multicenter, comparative effectiveness or translational clinical trials. The decision to investigate the surgical treatment options for IMR has provided the opportunity to identify investigational issues that will be relevant to all future surgical clinical research.

It is clear that one element for success is the creation of a culture of surgical investigation that is dissociated from the treatment paradigms that have been developed in the absence of evidence from randomized clinical trials. The second element of success is a shared vision of cardiac surgical investigation with the cardiology community at each site, within each region, and nationally. The effort must begin with engaged and committed surgical leadership at each clinical site, coupled with diplomatic and persistent engagement of the cardiology community. The nature of a patient referral for surgery generally confers the expectation on the part of each participant that a specific procedure will be performed. Although expectations must be adjudicated at the local level, patients, surgical investigators, and their referring physicians must eventually be willing to embrace participation in a randomized trial to provide greater insight into best practices. No single formula works to achieve this goal; however, lessons have been learned through the CTSN that will help future trials.

One important lesson concerns enrollment. The core CTSN clinical sites were activated for enrollment as of 
April 2009. Initially, the accrual rate was substantially less than estimated by historical rates of combined CABG and mitral valve procedures at the clinical sites. An extensive evaluation of the protocol, the structure of the CTSN, and site performance characteristics was undertaken, including multidisciplinary visits to each site. This evaluation disclosed several obstacles and inefficiencies that were amenable to improvement by protocol modification and establishment of the clear expectation that each site develop a culture of clinical research to include surgeons and cardiologists in collaboration.

It became clear that the historical prevalence of IMR had been significantly overestimated through historical reliance on contrast ventriculography and qualitative TTE and TEE estimates of the degree of mitral regurgitation. A review of the Duke Cardiovascular Database revealed that patients often have a change in mitral regurgitation severity grade when evaluated by TEE in the operating room. In a series of 430 patients with both preoperative TTE and intraoperative TEE, $19 \%$ of patients with mild mitral regurgitation documented preoperatively were found to have moderate mitral regurgitation in the operating room. Among patients who had moderate mitral regurgitation preoperatively, $14 \%$ were found to have severe mitral regurgitation in the operating room. The clinical decision making in these cases was undoubtedly driven by the intraoperative TEE findings; however, such patients would not be eligible for this trial. The more common occurrence of a reduction in the severity of mitral regurgitation seen on intraoperative TEE (37\% of cases of preoperative moderate mitral regurgitation determined intraoperatively to be mild) does not prevent enrollment in the protocol as designed, because the qualifying echocardiogram is the preoperative TTE study. The protocol development committee, in collaboration with the echocardiographic core laboratory leadership, therefore recommended that the determination of degree of mitral regurgitation be made by the site echocardiographer from the TTE according to all the primary and secondary criteria outlined in Tables 1 and 2.

Site visits with surgeons, cardiologists, and institutional leadership, as well as reviews of screening logs and meetings of the CTSN steering committee, provided new insights into the interactions among patients, referring cardiologists, site cardiologists, and surgeons that influence trial conduct and enrollment. It became clear that the cardiovascular community has developed paradigms for the management of patients with IMR that are not evidence based but could affect patient recruitment.

Treatment of patients with coronary artery disease and IMR has generally related to a qualitative assessment of the severity of mitral regurgitation, the presence of heart failure symptoms, and the correlation of coronary artery disease with myocardial ischemia, viability, and function. In the setting of moderate IMR, treatment paradigms are easily biased and may in some instances lead to a premature recommendation for mitral valve surgery that can preclude randomization into the trial once the expectation for repair has been established in the patient's mind. The generalizability of the study results may also be negatively affected if only patients with IMR toward the lower end of the moderate scale are enrolled. Similar concerns have been raised regarding patient recruitment in trials of percutaneous patent foramen ovale closure. CTSN cardiologists and surgeons realized that joint evidence-based decision making and clinical equipoise would be required to enable patient enrollment.

The dynamic nature of IMR was also found to be a major impediment to patient enrollment. Clinically indicated TTE studies, performed with variable image acquisition, were frequently found to demonstrate suitable IMR severity but failed to capture either the primary end point measure (LVESVI) or the ERO area. Subsequent research TTE studies, performed under the conditions of optimal medical therapy for ischemia and after diuresis, frequently then failed to meet the criteria for mitral regurgitation severity. Thus although such patients had the appropriate substrate for enrollment in the trial and underwent treatment for IMR of the severity seen on presentation, they were not eligible for randomization. This finding led to site-specific improvements in the quality of clinical TTE, thus enabling capture of the key elements of IMR in patients at initial presentation.

A systematic review of screening failures also revealed several limiting exclusion criteria, including previous cardiac surgery, planned concomitant surgical correction of atrial fibrillation, and a rigid threshold defining exclusion for pulmonary hypertension regardless of its relationship to the degree of mitral regurgitation. Revisions to the eligibility criteria were approved by the data and safety monitoring board in May 2009.

After this intensive 2-month period of self-evaluation and modifications to the protocol, a new target accrual rate (12.4 patients per month) was established. The current enrollment rate meets expectations. In addition, criteria were established within the CTSN for the incorporation of ancillary and satellite sites, and 12 additional sites have been activated. As of this writing, the Surgical Interventions for Moderate Ischemic Mitral Regurgitation Trial has enrolled 144 patients, or $48 \%$ percent of the target study population.

The process of creating and implementing an effective investigational network to address significant cardiac surgical problems has been enlightening. The design of this trial, and our experience in conducting it, has revealed limitations in the application of observational data to patient care. The absence of robust randomized trial evidence regarding the treatment of IMR has severely limited the ability of surgeons and cardiologists to determine the best options for these patients. 


\section{References}

1. Izumi S, Miyatake K, Beppu S, Park YD, Nagata S, Kinoshita N, et al. Mechanism of mitral regurgitation in patients with myocardial infarction: a study using real-time two-dimensional Doppler flow imaging and echocardiography. Circulation. 1987;76:777-85.

2. Kono T, Sabbah NH, Rosman H, Alam M, Jafri S, Goldstein S. Left ventricular shape is the primary determinant of functional mitral regurgitation in heart failure. J Am Coll Cardiol. 1992;20:1594-8.

3. Lamas GA, Mitchell GF, Flaker GC, Smith SC Jr, Gersh BJ, Basta L, et al. Clinical significance of mitral regurgitation after acute myocardial infarction. Survival and Ventricular Enlargement Investigators. Circulation. 1997;96:827-33.

4. Ellis SG, Whitlow PL, Raymond RE, Schneider JP. Impact of mitral regurgitation on long-term survival after percutaneous coronary intervention. Am J Cardiol. 2002;89:315-8.

5. Adler D, Goldman L, O’Neil A, Cook EF, Mudge GH Jr, Shemin RJ, et al. Longterm survival of more than 2,000 patients after coronary artery bypass grafting. Am J Cardiol. 1986;58:195-202.

6. Bursi F, Enriquez-Sarano M, Nkomo VT, Jacobsen SJ, Weston SA, Meverden RA, et al. Heart failure and death after myocardial infarction in the community: the emerging role of mitral regurgitation. Circulation. 2005;111:295-301.

7. Schroder JN, Williams ML, Hata JA, Muhlbaier LH, Swaminathan M, Mathew JP, et al. Impact of mitral valve regurgitation evaluated by intraoperative transesophageal echocardiography on long-term outcomes after coronary artery bypass grafting. Circulation. 2005;112(9 Suppl):I293-8.

8. Kang DH, Kim MJ, Kang SJ, Song JM, Song H, Hong MK, et al. Mitral valve repair versus revascularization alone in the treatment of ischemic mitral regurgitation. Circulation. 2006;114(1 Suppl):I499-503.

9. Gillinov AM, Wierup PN, Blackstone EH, Bishay ES, Cosgrove DM, White J, et al. Is repair preferable to replacement for ischemic mitral regurgitation? J Thorac Cardiovasc Surg. 2001;122:125-41.

10. Aklog L, Filsoufi F, Flores KQ, Chen RH, Cohn LH, Nathan NS, et al. Does coronary artery bypass grafting alone correct moderate ischemic mitral regurgitation? Circulation. 2001;104(12 Suppl 1):I68-75.

11. Trichon BH, Glower DD, Shaw LK, Cabell CH, Anstrom KJ, Felker GM, et al. Survival after coronary revascularization, with and without mitral valve surgery, in patients with ischemic mitral regurgitation. Circulation. 2003;108(Suppl 1): II103-10.
12. Fattouch K, Guccione F, Sampognaro R, Panzarella G, Corrado E, Navarra E, et al. POINT: Efficacy of adding mitral valve restrictive annuloplasty to coronary artery bypass grafting in patients with moderate ischemic mitral valve regurgitation: a randomized trial. J Thorac Cardiovasc Surg. 2009;138:278-85.

13. Fattouch K, Sampognaro R, Speziale G, Salardino M, Novo G, Caruso M, et al. Impact of moderate ischemic mitral regurgitation after isolated coronary artery bypass grafting. Ann Thorac Surg. 2010;90:1187-94.

14. Balu V, Hershowitz S, Zaki Masud AR, Bhayana JN, Dean DC. Mitral regurgitation in coronary artery disease. Chest. 1982;81:550-5.

15. Christenson JT, Simonet F, Bloch A, Maurice J, Velebit V, Schmuziger M. Should a mild to moderate ischemic mitral valve regurgitation in patients with poor left ventricular function be repaired or not? J Heart Valve Dis. 1995;4:484-9.

16. Grigioni F, Detaint D, Avierinos J, Scott C, Tajik J, Enriquez-Sarano M Contribution of ischemic mitral regurgitation to congestive heart failure after myocardial infarction. J Am Coll Cardiol. 2005;45:260-7.

17. American College of Cardiology/American Heart Association Task Force on Practice Guidelines; Society of Cardiovascular Anesthesiologists; Society for Cardiovascular Angiography and Interventions; Society of Thoracic Surgeons; Bonow RO, Carabello BA, et al. ACC/AHA 2006 guidelines for the management of patients with valvular heart disease: a report of the American College of Cardiology/American Heart Association Task Force on Practice Guidelines (writing committee to revise the 1998 Guidelines for the Management of Patients With Valvular Heart Disease): developed in collaboration with the Society of Cardiovascular Anesthesiologists: endorsed by the Society for Cardiovascular Angiography and Interventions and the Society of Thoracic Surgeons. Circulation. 2006;114:e84-231.

18. Baumgartner WA, Burrows S, del Nido PJ, Gardner TJ, Goldberg S, Gorman RC et al. Recommendations of the National Heart, Lung, and Blood Institute Working Group on Future Direction in Cardiac Surgery. Circulation. 2005;111 3007-13.

19. Enriquez-Sarano M, Avierinos JF, Messika-Zeitoun D, Detaint D, Capps M, Nkomo V, et al. Quantitative determinants of the outcome of asymptomatic mitral regurgitation. N Engl J Med. 2005;352:875-83.

20. Zoghbi WA, Enriquez-Sarano M, Foster E, Grayburn PA, Kraft CD, Levine RA et al. Recommendations for the evaluation of the severity of native valvular regurgitation with two-dimensional and Doppler echocardiography. J Am Soc Echocardiogr. 2003;16:777-802. 


\section{APPENDIX E1. CARDIOTHORACIC SURGICAL TRIALS NETWORK (CTSN) \\ National Heart, Lung and Blood Institute}

Marissa A. Miller, Wendy C. Taddei-Peters, Dennis Buxton, Ron Caulder, Nancy L. Geller, David Gordon, Neal O. Jeffries, Albert Lee

National Institute of Neurological Disorders and Stroke. Claudia S. Moy

Canadian Institutes of Health Research. Ilana Kogan Gombos, Jennifer Ralph

Network chairs. Christiana Care Health System, Timothy J. Gardner (chair); Brigham and Women's Hospital, Patrick T. O'Gara (cochair)

Data coordinating center. International Center for Health Outcomes and Innovation Research (InCHOIR) in the Department of Health Evidence and Policy, Mount Sinai School of Medicine, Annetine C. Gelijns, Michael K. Parides, Deborah D. Ascheim, Alan J. Moskowitz, Ellen Moquete, Eric A. Rose, Melissa Chase, Yingchun Chen, Rosemarie Gagliardi, Alejandra Guerchicoff, Lopa Gupta, Alexander Iribarne, Edlira Kumbarce, Angela Lee, Ron Levitan, Karen O'Sullivan, Mark J. Russo, Milerva Santos, William Slavik, Alan Weinberg, Martin Wells, Paula Williams, Carrie Wood, Xia Ye

Core clinical site investigators. Cleveland Clinic Foundation, Eugene H. Blackstone (principal investigator), A. Marc Gillinov, Tomislav Mihaljevic, Richard A. Grimm, Ben Barzilai, Bruce D. Lindsay, Christine Whitman, Denise Kosty Sweeney, Roberta Palumbo, NHLBI Clinical Research Scholars Gregory Pattakos and Pamela A. Clarke; Columbia University, Michael Argenziano (principal investigator), Mathew Williams, Lyn Goldsmith, Rebecca T. Hahn, Linda Gillam, Craig R. Smith, Yoshifumi Naka, Allan Stewart, Allan Schwartz; Duke University, Peter K. Smith (principal investigator), Stacey Welsh, John H. Alexander, Carmelo A. Milano, Donald D. Glower, Joseph P. Mathew, J. Kevin Harrison, NHLBI Clinical Research Scholars Mark F. Berry, Cyrus J. Parsa, Betty C. Tong, and Judson B. Williams; East Carolina Heart Institute, T. Bruce Ferguson (principal investigator), Alan P. Kypson, Evelio Rodriguez, Malissa Harris, Brenda Akers, Allison O'Neal; Emory University, John D. Puskas (principal investigator), Vinod H. Thourani, Robert Guyton, Jefferson Baer, Kim Baio, Alexis Neill; Montefiore Einstein Heart Center, Robert E. Michler (principal investigator), Ricardo Bello, David A. D'Alessandro, Joseph J. DeRose, Jr, Daniel J. Goldstein, Cynthia Taub, Daniel Spevak, Roger Swayze; Montreal Heart Institute, Louis P. Perrault (principal investigator), Arsène-Joseph Basmadjian, Denis Bouchard, Michel Carrier, Raymond Cartier, Michel Pellerin, Sophie Robichaud; NIH Heart Center at Suburban Hospital, Keith A. Horvath (principal investigator), Philip C. Corcoran, Michael P. Siegenthaler, Mandy Murphy, Margaret Iraola, Ann
Greenberg; University of Pennsylvania, Michael A. Acker (principle investigator), Y. Joseph Woo, Wilson Y. Szeto, Mary Lou Mayer; University of Virginia, Irving L. Kron (principal investigator), Gorav Ailawadi, Karen Johnston, John M. Dent, Sandra Burks, Kim Gahring

Affiliate/ancillary sites. Hôpital du Sacré-Cœur de Montréal, Pierre Pagé (principal investigator), Hugues Jeanmart, Philippe Demers, Claude Sauvé, Carole Sirois; Institut Universitaire de Cardiologie et de Pneumologie de Québec, Pierre Voisine (principal investigator), François Dagenais, Eric Dumont, Gladys Dussault; Inova Fairfax Hospital, Alan M. Speir (principal investigator) Niv Ad, Minh Dang; The Ohio State University Medical Center, Chittoor B. Sai-Sudhakar (principal investigator), Danielle Jones; WellStar Health System, Kennestone Hospital, William A. Cooper (principal investigator), Rajnish Prasad, Richard J. Myung, Jennifer LaCorte, Melinda Mock

Satellite sites. Baylor Research Institute, Michael J. Mack (principal investigator), Robert Smith, William Ryan, Jennifer Withers; Brigham and Women's Hospital, Frederick Y. Chen (principal investigator), R. Morton Bolman III, Anne M. Burgess, Debra Conboy; Jewish and St. Mary's Hospital, Mark S. Slaughter (principal investigator), Matthew Williams, Marcus Stoddard, Heather Moody; Mission Hospital, Mark A. Groh (principal investigator), Ben Trichon, Todd Hansen, Claudine Cuento; University of Southern California, Vaughn A. Starnes (principal investigator), Michael Bowdish, Becky Lopez; University of Maryland, James S. Gammie (principal investigator), Mandeep Mehra, Bartley Griffith, Dana Beach; Washington University, Ralph J. Damiano, Jr (principal investigator), Scott Silvestry, Marc Moon, Jennifer Lawton

Cardiopulmonary exercise core laboratory. Henry Ford Hospital, Steven J. Keteyian, Clinton A. Brawner

Echocardiography core laboratory. Massachusetts General Hospital, Judy Hung, Xin Zeng

Electrophysiology core laboratory. University of Rochester Medical Center, Jean-Philippe Couderc

Neurocognitive core laboratory. Duke University, Joseph P. Mathew

Protocol review committee. David A. Bull (chair), Patrice Desvigne-Nickens (executive secretary), Dennis O. Dixon, Mark Haigney, Richard Holubkov, Alice Jacobs, Frank Miller, John M. Murkin, John Spertus, Andrew S. Wechsler Data and safety monitoring board. Frank Sellke (chair), Cheryl L. McDonald (executive secretary), Robert Byington, Neal Dickert, Dennis O. Dixon, John S. Ikonomidis, David O. Williams, Clyde W. Yancy

Medical monitors. James C. Fang, Wayne Richenbacher Overall event adjudication committee. Vivek Rao (chair), Karen L. Furie, Rachel Miller, Sean Pinney, William C. Roberts Infection event adjudication committee. Rachel Miller (chair), Shirish Huprikar, Marilyn Levi 